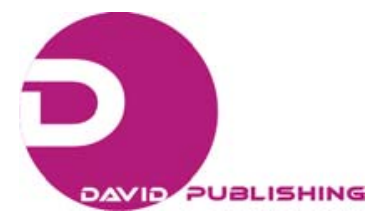

\title{
Electro-Optic Modulation in a Sub-Wavelength Gap-Plasmon Guide
}

Subimal Deb, Sarat Kumar Kuruhuru, Anantha Lakshmi Pochincherla and Subhasish Dutta Gupta

School of Physics, University of Hyderabad, Hyderabad, Andhra Pradesh 500046, India

Received: March 04, 2010 / Accepted: March 29, 2010 / Published: February 10, 2011.

\begin{abstract}
The efficient electro-optic modulation in a sub-wavelength gap-plasmon waveguide (GPW) was formed by an electro-optic polymer with metal coatings. The proposed device is studied in the attenuated total reflection (using the characteristic matrix method) and end-fire configurations (using the finite difference time domain method). In dealing with the end-fire configuration we used a taper from a micron sized guide to the GPW. The structure is shown to exhibit large phase accumulation over short distances, controllable by the applied modulating voltage.
\end{abstract}

Key words: Electro-optic modulation, gap plasmon waveguide, electro-optic polymer.

\section{Introduction}

Transport, confinement and modulation of electromagnetic energy and miniaturization of optoelectronic devices have been the focus of research over the past few years $[1,2]$. The goal has been to beat the Rayleigh limit and to improve the performance of components like inter/intra chip optical interconnects, low loss waveguides, light emitting devices, modulators and amplifiers for high speed circuits and optical signal processing [3]. For many of such applications modulation of signals is crucial and requires control over the optical properties, namely, refractive index, of the constituent parts. Thermo-optic, acousto-optic and electro-optic materials have thus been probed for use in intensity and phase modulators [3, 4]. Novel materials are being probed in the context of easy integration with electronic circuits and novel chip designs $[5,6]$. The weak electro-optical properties of silicon have been used in micrometer-sized Mach-Zehnder and ring resonator-based modulators [5, 7, 8]. Electro-optic

Corresponding author: Subimal Deb (1981- ), male, Ph.D., research associate, research field: photonics. E-mail: subimal. deb@gmail.com. polymers have been studied in detail [6] in micron-sized multilayered structures of poled polymer on glass substrate coated with transparent conducting oxide [9]. Further reduction in the device size requires confinement of electromagnetic energy to sub-wavelength dimensions, posing a challenging problem due to the Rayleigh criterion. It is known that lateral confinement of the modes in sub-wavelength guides using photonic bandgap structures or conventional waveguides is not possible [10]. Metal-dielectric guiding structures supporting surface plasmons and array of metal nano particles, each supporting localized plasmons, have been exploited to this end [11]. The major differences between these geometries have been discussed in detail by Ozbay [11]. A recent work showed the free-space coupling of incident radiation and modulation of reflected light [12]. The design had a planar geometry of nanometer-sized gold and micron-sized electro-optic polymer layers. Efficient confinement and transportation of light within sub-wavelength dimensions in the microwave and visible regime using arrays of copper rods and gold nanoparticles, respectively, have been demonstrated $[10,13]$. An 
efficient and highly reproducible sub-wavelength scale resonator made of $590 \mathrm{~nm}$ long ridge on a single crystal $\mathrm{Au}$ substrate with surface plasmon polariton modes having a diameter less than $100 \mathrm{~nm}$ were shown [14]. A numerical study of a $50 \mathrm{~nm}$ wide silver-cladded air cavity resonator coupled to lateral or inserted cavities capable of functioning as filters for plasmonic waves has been reported [15]. Efficient propagation in surface plasmon polariton band gap structure of $45 \mathrm{~nm}$ thick gold film on glass substrate at near-optical wavelengths was shown [16]. Splitting and combination of line defect modes with the same corrugated gold film surface over $20 \mu \mathrm{m}$ long Y-junction was observed. Bend loss analysis indicates their feasibility for photonic circuits. It is now well understood that one of the coupled surface modes in a thin metal film (long range surface plasmon (LRSP)), is associated with larger Q-factor and field enhancement. Some of the recent studies exploit such properties of LRSP to achieve modulation [17]. A Mach-Zehnder interferometer based structure exploiting LRSP modes was studied at telecommunication wavelengths [4]. It consisted of a $15 \mathrm{~nm}$ thick gold stripe embedded in a thermo-optic polymer and showed efficient modulation and switching properties. LRSP waveguides have been fabricated using a direct wafer bonding method [18]. The structure consisted of a $20 \mathrm{~nm}$ thick gold stripe embedded inside a $32 \mu \mathrm{m}$ thick $\mathrm{LiNbO}_{3}$ stack. Electro-optic measurements at a wavelength of $1.55 \mu \mathrm{m}$ showed that the cladding retains bulk properties even at such small dimensions. Another promising candidate for achieving electro-optic modulation (EOM) in sub-wavelength structures is nanowire devices. Broadband intensity modulation with high modulation depths were proposed in studies of $\sim 100 \mathrm{~nm}$ diameter $\mathrm{CdS}$ and GaN nanowires at optical wavelengths $[19,20]$. Efficient energy confinement and feasibility of short range interconnections with a plasmonic guide were shown both experimentally and by numerical computation [2, 13, 21]. These studies suggest feasibility of using polymers for efficient modulators due to their ease of integration with several passive materials.

In this paper, we concentrate on a planar geometry of metal-dielectric-metal guides (known as gap-plasmon guides (GPW)) with sub-wavelength lateral dimension in order to show efficient EOM. It is known that well confined plasmonic modes in the GPW geometry have longer propagation lengths [22]. We use the plasmon single-mode regime of such a guide with an electro-optic polymer between the two metal plates. It was shown earlier how the modes of the GPW could be used for slowing down light [23]. Here we demonstrate how the same modes can, in principle, be used for achieving EOM in attenuated total reflection (ATR) or end-fire configuration. We show that the device is expected to lead to a large accumulation of phase. Moreover, this phase is shown to be controllable by the applied modulating voltage via the refractive index dependence of the electro-optic polymer on the electric field. The end-fire configuration is particularly interesting since in all probability, such is the way a guide is to be excited. However, focusing to a spot size less than 50 $\mathrm{nm}$ may pose a challenging problem. Besides, the modulator may form a part of a usual communication system. Keeping in view the aforementioned we use a standard $1.25 \mu \mathrm{m}$ guide (a model for the fiber) followed by a tapered region to the $50 \mathrm{~nm}$ guide (Fig. 1). Recently such a structure was investigated to probe the loss in the micro-to-nano interfacing and a loss of

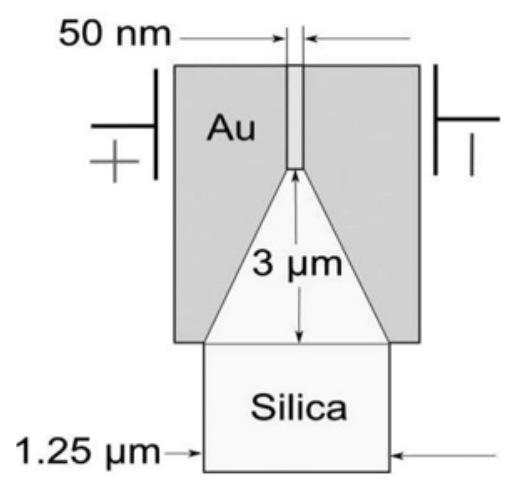

Fig. 1 Schematic of the proposed GPW structure. 
about $30 \%$ was reported [24]. Clearly, the analytical treatment could be too cumbersome. Hence we resort to numerical finite difference time domain (FDTD) scheme [25] (using the commercial product Fullwave [26]) to analyze such a structure. We assume the polymer to be isotropic and the structure to have a zero insertion loss.

The structure of the paper is as follows. In section 2 we show how the sensitivity of the surface plasmon resonance on the change of the core refractive index (via the electro-optic effect) can be used for intensity-modulation in reflection in ATR geometry. The next section 3 deals with the FDTD solution for the end-fire configuration. We summarize the important results in Conclusions.

\section{Electro-Optic Modulation in the ATR Geometry}

It is now well understood that the modes of a planar metal-dielectric structure can be studied in the attenuated total reflection geometry, wherein the guiding structure is loaded on top by a high index prism (Fig. 2). The excitation of the guided and surface modes can be read out in the dips of the reflection coefficient for specific angles of incidence. The location of the dips is extremely sensitive to the guide parameters, the ambient medium refractive index and on the surface conditions. This sensitivity has been exploited to a great deal to realize plasmonic and waveguide sensors and electro-optic modulators [27]. In this section, we use the specifics of our GPW to show that similar ideas also hold (as expected) at sub-wavelength scales where the guide core width is reduced to about $50 \mathrm{~nm}$. Our GPW structure consists of an electro-optic polymer of width $\mathrm{d}_{2}$ sandwiched between two identical gold layers of width $d_{1}$ (Fig. 2). The polymer is assumed to be isotropic for simplicity and therefore the change in dielectric permittivity $(\varepsilon)$ can be written as [28]:

$$
\Delta \varepsilon=-\varepsilon^{2} \mathrm{r} \mathrm{E}
$$

where $r$ is the electro-optic coefficient, $\mathrm{E}$ is the applied electric field. We can further assume a flat response of

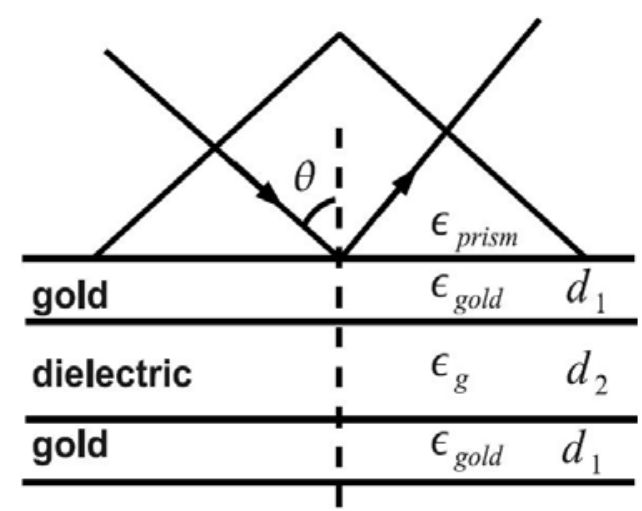

Fig. 2 ATR geometry for exciting the gap plasmon structure.
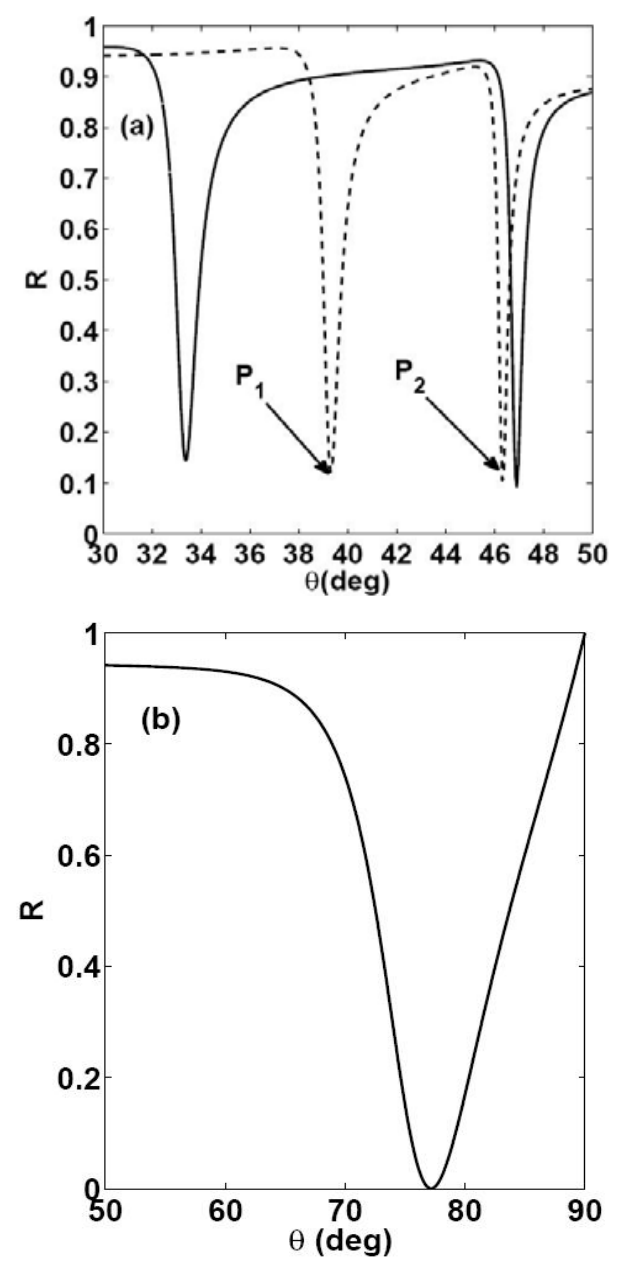

Fig. 3 (a) The guided (plasmonic) mode marked $P_{1}\left(P_{2}\right)$ shifts to lower (higher) effective index (i.e., smaller angle of incidence $\theta$ ) when the $d_{2}$ is decreased from $0.8 \mu \mathrm{m}$ (dashed) to $0.6 \mu \mathrm{m}$ (solid), (b) Only a plasmonic mode is excited at $d_{2}$ $=50 \mathrm{~nm}$ (below the cut-off for the guided modes). All plots correspond to TM polarization.

$\varepsilon$ over the range of working frequencies leading to

$$
\varepsilon=\varepsilon_{\mathrm{g}}\left(1-\varepsilon_{\mathrm{g}} \mathrm{r} \mathrm{E}\right)=\varepsilon_{\mathrm{g}}\left(1-\mathrm{E}^{\prime}\right)
$$


Thus the permittivity of the electro-optic polymer layer has been written down in terms of a dimensionless electric field amplitude $\left(\mathrm{E}^{\prime}=\varepsilon_{\mathrm{g}} \mathrm{rE}\right)$ where $\varepsilon_{\mathrm{g}}$ is the permittivity of the polymer layer without any applied voltage.

The structure is excited by a monochromatic plane wave incident through a high index prism (refractive index $n_{i}$ ) at an angle $\theta$. The modes of such a GPW were studied in detail $[23,24]$. Below a certain cut-off the only mode that can survive in the structure is the plasmon mode, which can be excited only with p-polarized light. Thus such a guide does not support (at about $\mathrm{d}_{2}=50 \mathrm{~nm}$ ) any s-polarized waves and essentially is a single mode device below this cut-off width at $\lambda=1.55 \mu \mathrm{m}$. The calculation of the refraction coefficient for such layered media is fairly standard and we use the characteristic matrix technique to arrive at the magnitude of the intensity reflection coefficient $R$. The results for $R$ as a function of the angle of incidence for applied field $\mathrm{E}^{\prime}=0$ is shown in Fig. 3 for several values of the guide core thickness (a) $0.8 \mu \mathrm{m}$ (dashed), $0.6 \mu \mathrm{m}$ (solid) and (b) $50 \mathrm{~nm}$. Other parameters are as follows: dielectric permittivities of the polymer guide, gold and high-index prism are $\varepsilon_{\mathrm{g}}=$ $3, \varepsilon_{\text {gold }}=-132+12.6 \mathrm{i}$ and $\varepsilon_{\text {prism }}=6.145$, respectively and cladding thickness $\mathrm{d}_{1}=30 \mathrm{~nm}$.

Then the change in the mode pattern due to the applied voltage and the corresponding change in the reflectivity have been studied. It is clear that with a change in the refractive index due to the change in the applied voltage, the resonance dip will get shifted. The effective index $\left(\mathrm{n}_{\text {eff }}\right)$ of a particular mode can be extracted by using the following relation

$$
\mathrm{n}_{\text {eff }} \sin \theta_{\text {mode }}=n_{\mathrm{i}} \sin \theta_{\mathrm{i}}
$$

where $\theta_{\text {mode }}$ is the value of the angle of incidence where the dip occurs. The results for the effective index as a function of the normalized dc voltage are shown in Fig. 4a. It is clear that an increase in the voltage reduces the core refractive index and hence the effective index. A $10 \%$ change in $\varepsilon$ caused by the change in the voltage brings about a corresponding
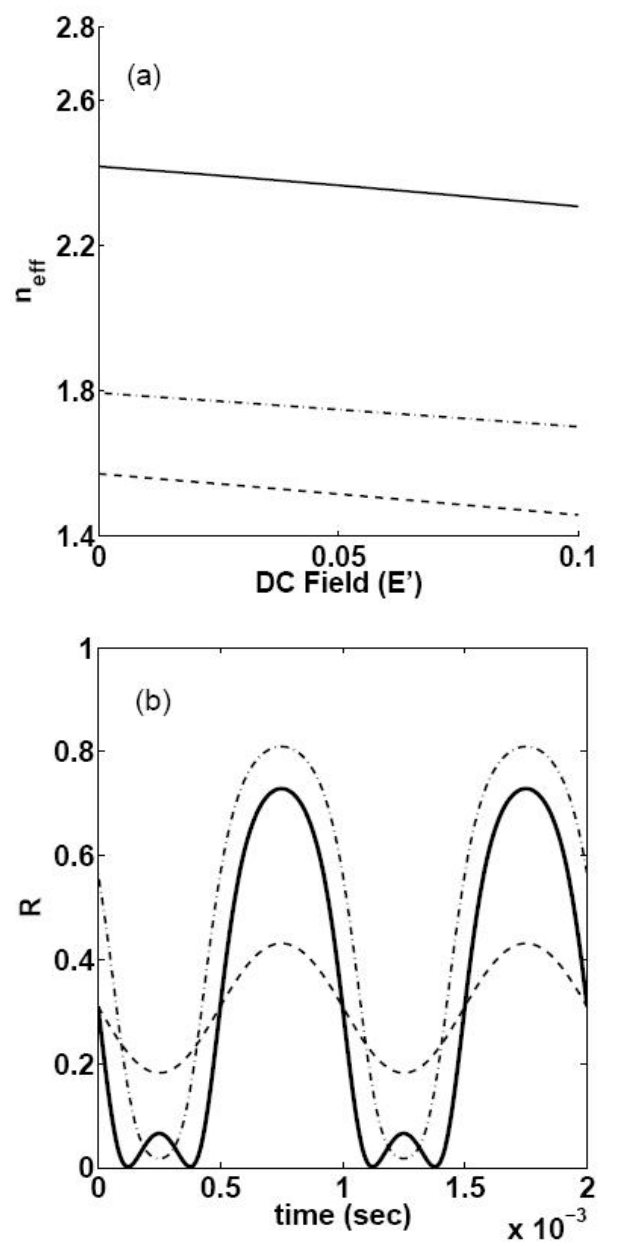

Fig. 4 (a) $n_{\text {eff }}$ as a function of the applied field $E$ '. The lowest (middle) curve corresponds to the mode marked $P_{1}$ $\left(P_{2}\right)$ in Fig. 3a at $d_{2}=0.8 \mu \mathrm{m}$. The upper (solid) curve corresponds to the plasmonic mode for $d_{2}=50 \mathrm{~nm}$, (b) the ac response at $d_{2}=50 \mathrm{~nm}$ with $E^{\prime}=E_{0} \sin 2 \pi \mathrm{ft}$ at $1 \mathrm{kHz}$. Reflectivity of the GPW structure for $E^{\prime}=0.05$ at $\theta=$ 73.88 (solid), 72 (dash-dot) and for $E^{\prime}=0.01$ at $\theta=$ 73.88 (dashed).

change in the effective index.

The effect of an ac voltage on the intensity reflection can be dramatic, since such a voltage can take the systems to off resonance and again bring it back to resonance. This results in an oscillatory response in the reflectivity of Fig. $4 \mathrm{~b}$. The large values of reflectivity correspond to the case where the mode is not excited efficiently, while a low value implies efficient excitation of the guided/plasmon modes. These upper and lower bounds can be regulated by choosing the detuning from the surface plasmon 
resonance. One can thus regulate the efficiency of excitation of the guided/plasmon mode. These features are shown in Fig. $4 b$, for $d_{2}=50 \mathrm{~nm}$ with $E^{\prime}=E_{0} \sin$ $2 \pi \mathrm{ft}$ for $\mathrm{f}=1 \mathrm{kHz}$. We show such modulation in Fig. $4 \mathrm{~b}$ over two cycles of the applied ac field. We show this for two amplitudes of the applied field, namely, $\mathrm{E}_{0}=0.01$ and 0.05. At $\mathrm{E}_{0}=0.05, \theta=73.88^{\circ}$ the plasmonic mode is efficiently coupled into the sub-wavelength guide, that is, a good modulation depth is achieved. With $\mathrm{E}_{0}=0.05$, changing $\theta$ to $72^{\circ}$ (dashed) decreases the modulation depth as is clear from the figure.

\section{Phase Modulation in the End-Fire Configuration}

Our GPW structure, in practice, need to be interfaced with other components in an optical circuit. It is thus necessary to analyze our structure for the end-fire excitation. We consider coupling of the polymer guide to a standard $1.25 \mu \mathrm{m}$ silica guide through a taper of height $3 \mu \mathrm{m}$. As mentioned earlier, such structures were analyzed in detail for the loss in the tapered region [24]. It was shown that about $70 \%$ of the incident flux can be coupled into the nano-guide. We study a similar system except that the core is now replaced by the electro-optic polymer.

To be specific, we use the FDTD technique to study the coupling of electromagnetic energy from the micro-guide to the $50 \mathrm{~nm}$ GPW. The simulation again confirms that only the TM polarized modes of the micro-guide can be coupled to the sub-wavelength GPW (Fig. 5). The phase accumulated by the plasmon mode propagating along the polymer channel is expected to be proportional to the applied modulating voltage for small variations of $\varepsilon$ from $\varepsilon_{\mathrm{g}}$ vide Eq. (2). Further, since the electric field in the channel is inversely proportional to the distance between the electrodes, small variations in the applied voltage (amounting to small power consumptions by the circuit) should be sufficient to produce the requisite variations of $\varepsilon$ in the sub-wavelength GPW. We extracted

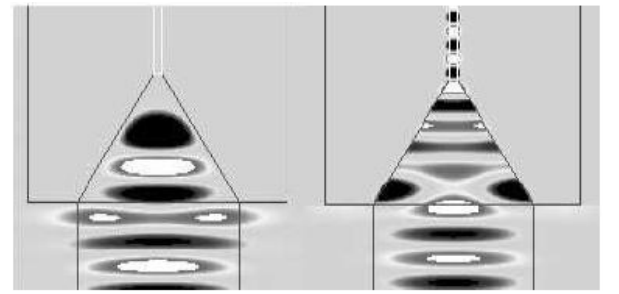

Fig. 5 FDTD simulation of propagation of TE (left) and TM (right) polarized waves through the structure. TE waves are reflected back whereas the TM waves can be coupled into the nano-structure.

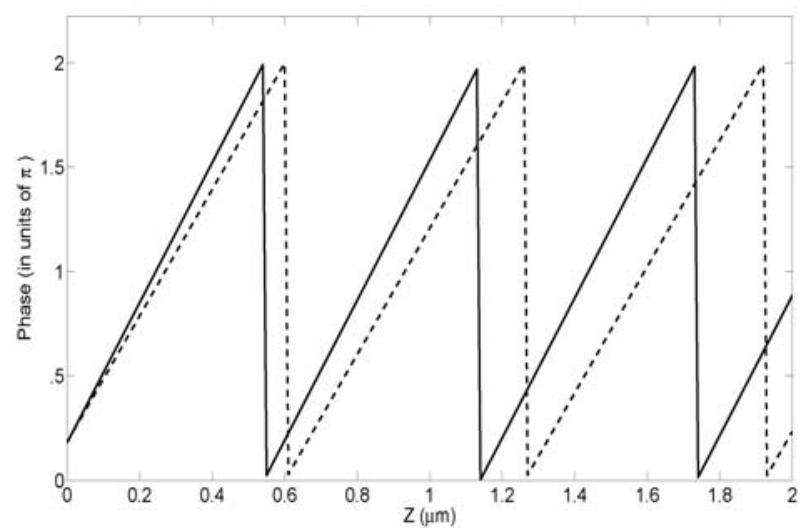

Fig. 6 The phase (modulo $2 \pi$ ) of the magnetic field component at the centre of the guide measured along the propagation direction for $\mathrm{E}^{\prime}=\mathbf{0 . 1}$ (dashed) and $\mathbf{- 0 . 1}$ (solid).

the phase of the magnetic field in the plasmon mode as a function of the propagation distance for two voltages corresponding to $\mathrm{E}^{\prime}= \pm 0.1$ in the simulation (Fig. 6). The calculation of the accumulated phase of the plasmon mode gives a phase difference of about $\pi / 2$ for the two values of $E$ ' at a propagation length of $1.54 \mu \mathrm{m}$. Thus it is shown that, in principle, the GPW configuration can be used effectively as a phase modulator over short distances by controlling the applied field.

\section{Conclusions}

The feasibility of a sub-wavelength GPW as an efficient electro-optic intensity and phase modulator has been demonstrated by exploiting the single mode regime of the guide, albeit with the assumptions of an isotropic polymer and zero insertion loss. This proposal in principle, demonstrates the possibility of achieving electro-optic modulation at sub-wavelength scales and may find applications in nano-scale integrated 
optical circuits.

\section{Acknowledgments}

The authors are thankful to the Nano Initiative Programme of the University of Hyderabad. One of the authors (SDG) is also thankful to the Department of Science and Technology, Govt. of India for partial support.

\section{References}

[1] W.L. Barnes, A. Dereux, T.W. Ebbesen, Surface plasmon subwavelength optics, Nature 424 (2003) 824-830.

[2] H.A. Atwater, The promise of plasmonics, Sci. Am. 4 (2007) 56-63.

[3] R.G. Hunsperger, Integrated Optics, 6th ed., Springer, 2009.

[4] T. Nikolajsen, K. Leosson, S.I. Bozhevolnyi, Surface plasmon polariton based modulators and switches operating at telecom wavelengths, App. Phys. Lett. 85 (2004) 5833-5835.

[5] Q. Xu, B. Schmidt, S. Pradhan, M. Lipson, Micrometre-scale silicon electro-optic modulator, Nature 435 (2005) 325-327.

[6] R.M. de Ridder, A. Driessen, E. Rikkers, P.V. Lambeck, M.B.J. Diemeer, Design and fabrication of electro-optic polymer modulators and switches, Optical Materials 12 (1999) 205-214.

[7] R.S. Jacobsen, K.N. Andersen, P.I. Borel, J. Fage-Pedersen, L.H. Frandsen, O. Hansen, M. Kristensen, A.V. Lavrinenko, G. Moulin, H. Ou, C. Peucheret, B. Zsigri, A. Bjarklev, Strained silicon as a new electro-optic material, Nature 441 (2006) 199-202.

[8] C.A. Barrios, M. Lipson, Modeling and analysis of high-speed electro-optic modulation in high confinement silicon waveguides using metal-oxide-semiconductor configuration, J. App. Phys. 96 (2004) 6008-6015.

[9] D.H. Park, C.H. Lee, W.N. Herman, Analysis of multiple reflection effects in reflective measurements of electro-optic coefficients of poled polymers in multilayer structures, Optics Express 14 (2006) 8866-8884.

[10] S.A. Maier, P.G. Kik, H.A. Atwater, S. Meltzer, E. Harel, B.E. Koel, A.A.G. Requicha, Local detection of electromagnetic energy transport below the diffraction limit in metal nanoparticle plasmon waveguides, Nature Materials 2 (2003) 229-232.

[11] E. Ozbay, Plasmonics: Merging photonics and electronics at nanoscale dimensions, Science 311 (2006) 189-193.

[12] X. Deng, An electro-optic polymer modulator based on the free-space coupling technique, J. Opt. A: Pure Appl. Opt. 10 (2008) 015305.

[13] S.A. Maier, M.L. Brongersma, H.A. Atwater, Electromagnetic energy transport along arrays of closely spaced metal rods as an analogue to plasmonic devices, App. Phys. Lett. 78 (2001) 16-18.

[14] E.J.R. Vesseur, R. de Waele, H.J. Lezec, H.A. Atwater, F.J. García de Abajo, A. Polman, Surface plasmon polariton modes in a single-crystal $\mathrm{Au}$ nanoresonator fabricated using focused-ion-beam milling, App. Phys. Lett. 92 (2008) 083110.

[15] A. Noual, Y. Pennec, A. Akjouj, B. Djafari-Rouhani, L. Dobrzynski, Nanoscale plasmon waveguide including cavity resonator, J. Phys.: Condens. Matter. 21 (2009) 375301.

[16] S.I. Bozhevolnyi, V.S. Volkov, K. Leosson, A. Boltasseva, Bend loss in surface plasmon polariton band-gap structures, App. Phys. Lett. 79 (2001) 1076-1078.

[17] P. Berini, Long-range surface plasmon polaritons, Advances in Optics and Photonics 1 (2009) 484-588.

[18] P. Berini, G. Mattiussi, N. Lahoud, R. Charbonneau, Wafer-bonded surface plasmon waveguides, App. Phys. Lett. 90 (2007) 061108.

[19] C.J. Barrelet, A.B. Greytak, C.M. Lieber, Nanowire photonic circuit elements, Nano Letters 4 (2004) 1981-1985.

[20] A.B. Greytak, C.J. Barrelet, Y. Li, C.M. Lieber, Semiconductor nanowire laser and nanowire waveguide electro-optic modulators, App. Phys. Lett. 87 (2005) 151103.

[21] S.A. Maier, M.L. Brongersma, P.G. Kik, S. Meltzer, A.A.G. Requicha, B.E. Koel, H.A. Atwater, Plasmonics-a route to nanoscale optical devices, Advanced Materials 13 (2001) 1501-1505.

[22] S.I. Bozhevolnyi (Ed.), Plasmonic Nanoguides and Circuits, Pan Stanford Publishing, 2008.

[23] S. Dutta Gupta, Slow light with symmetric gap plasmon guides with finite width metal claddings, Pramana-Journal of Physics 72 (2009) 303-314.

[24] P. Ginzburg, D. Arbel, M. Orenstein, Gap plasmon polariton structure for very efficient microscale-tonanoscale interfacing, Opt. Lett. 31 (2006) 3288-3290.

[25] A. Taflove, S.C. Hagness, Computational Electrodynamics: The Finite-Difference Time-Domain Method, 3rd ed., Artech House, 2005.

[26] Available online at: http://www.rsoftdesign.com/products. php?sub=Component + Design\&itm=FullWAVE.

[27] T.P. Jannson, J.L. Jannson, B. Moslehi, High modulation rate optical plasmon waveguide modulator, US Patent No. 5,067,788 (1991).

[28] R.W. Boyd, Nonlinear Optics, 3rd ed., Academic Press, 2009. 